\title{
Upper-bounded no more: the exhaustive interpretation of non-strict comparison
}

\author{
Rick Nouwen
}

Published online: 26 November 2008

(C) The Author(s) 2008. This article is published with open access at Springerlink.com

\begin{abstract}
The paper concerns the expression of non-strict comparison, focusing in particular on constructions of the form $[n o(t) \ldots$.er than $]$ in modified numerals. The main empirical finding is the observation that negated comparatives contrast with regular comparatives in that the former but not the latter can give rise to (scalar) implicatures. It is shown that such a contrast falls out of theories of exhaustive interpretation that claim alternatives to form dense scales. An important result is that the paper sharpens the desiderata for theories of exhaustification.
\end{abstract}

Keywords Modified numerals · Exhaustive interpretation · Comparison

\section{Introduction}

The comparative morpheme, -er in English, expresses what I call strict comparison. That is, it corresponds to the $>$ - or $<$-relation rather than to the $\geq$ - or $\leq$-relation. In logic, the latter kind of comparison is related to the former kind by negation. That is, the $\geq$-relation is the complement of the $<$-relation and the $\leq$-relation is the complement of the $>$-relation. This paper considers to what extent these correspondences are fleshed out in natural language. My main focus is on a rather specific kind of comparative

Parts of this paper were presented at Sinn und Bedeutung 12 in Oslo. I am grateful to the audience for their comments and remarks. More in particular, I want to thank Jakub Dotlačil, Øystein Nilsen, Doris Penka, Hans-Christian Schmitz, and Magda Schwager for fruitful discussion. Thanks to Robert van Rooij for discussion during a presentation of this work in an earlier stage and to an anonymous reviewer for detailed and thought-provoking comments. This research was supported by a grant from the Netherlands Organisation for Scientific Research (NWO), which I gratefully acknowledge.

\section{R. Nouwen $(\triangle)$}

Utrecht Institute for Linguistics OTS, Utrecht University, Janskerkhof 13,

3512 BL, Utrecht, The Netherlands

e-mail: R.W.F.Nouwen@uu.nl 
construction, namely the kind that fits the scheme $[$ no $(t) \ldots$. . er $]$. In particular, I will be looking at the use of such constructions in modified numerals, as in (1).

Cody found no more than sixteen marbles.

The main reason for looking into the domain of modified numerals is because there we can compare the no more construction to other expressions that seem to involve non-strict comparison. Furthermore, as I will show, the present study sheds light on an important topic in the semantics of modified numerals, namely the issue of why they (generally) fail to give rise to implicatures. What I intend to show is that the mechanisms of exhaustive interpretation are sensitive to the distinction between strict and non-strict comparison.

A large part of the paper is devoted to establishing how exactly the data with respect to the interpretation of no(t) -er corresponds to basic assumptions regarding the semantics of comparison. Thus, Sect. 3 presents the observation that no more does not generally express non-strict comparison, but gives rise to stronger, doublebounded interpretations. I propose that these interpretations are due to (scalar) implicatures. In a nutshell, the observation is that an example like (1) is often interpreted not as expressing the complement of Cody finding more than 16 marbles, but rather as expressing that Cody found exactly 16 marbles, with an additional evaluative meaning according to which 16 is thought to be few. Such an interpretation, I will argue, can be accounted for if we assume that no more gets its expected logical interpretation, but that (under normal circumstances) this interpretation is subject to exhaustification, yielding a stronger interpretation, which is not only upper- but moreover lower-bounded. Section 4 discusses a theory of exhaustification from which these observations fall out straightforwardly, namely Fox and Hackl (2006). Section 5 discusses how the no more construction relates to other modifiers of numerals that have been thought to express non-strict comparison. I sum up the paper's main observations and the proposed account in Sect. 6 and give some suggestions for how to account for some additional aspects of the meaning of $n o(t)$-er. Finally, in Sect. 7, I explore and compare alternative explanations for the observed patterns.

First, in Sect. 2, I provide some necessary background on the relation between modified numerals, comparison, and implicatures.

\section{Background: modified numerals}

One of the most striking features of modified numerals is that they come in a large variety of forms, but (at least on first sight) carry only a limited range of meanings. If one assumes that the combination of a modifier and a numeral is a complex determiner (Barwise and Cooper 1981; Keenan and Stavi 1986), then it is tempting to think that the determiner denotation in (2a) is expressed by all the modified numerals in (2b). The same would hold for (3). 
(2) a. $\lambda A . \lambda B \cdot|A \cap B|>10$

b. more than ten

at least eleven

eleven or more

no fewer than eleven

(3) a. $\lambda A . \lambda B \cdot|A \cap B|<10$

b. less than ten

at most nine

nine or fewer

no more than nine

up to nine

It is becoming increasingly clear that such a view of the semantics of modified numerals is far too simplistic. In particular, there are three ways in which the picture sketched in (2) and (3) is wrong: (i) it is a mistake to believe that the expressions in (2b)/(3b) are semantically equivalent; (ii) it is a mistake to believe that the nature of the modifier (comparative, superlative, disjunction, negation, etc.) is immaterial to what the modifier expresses; and (iii) it is a mistake to believe that the semantics of any modified numeral is the relation in (2a) or (3a). I now roughly sketch how such conclusions follow from three important issues one finds in the recent literature on modified numerals. These issues will prove important for the discussion of no(t) -er below.

\subsection{Issue 1: No implicatures}

It has been observed on several occasions, but most explicitly in Krifka (1999), that contrary to what a naive semantics such as the one in $(2) /(3)$ predicts, modified numerals do not give rise to implicatures.

On the naive account, (4a) expresses (4b).

(4) a. Cody found more than ten marbles.

b. the number of marbles found by Cody $>10$

There are stronger alternatives to (4b) that line up on an entailment scale. That is, (4b) is asymmetrically entailed by the following:

(5) the number of marbles found by Cody $>11$

$\Leftarrow$ the number of marbles found by Cody $>12$

$\Leftarrow$ the number of marbles found by Cody $>13$

$\Leftarrow$ etc.

Through standard Gricean reasoning, one would expect an implicature to arise which denies these stronger alternatives. So (4a) should implicate that the number of marbles found by Cody does not exceed 11 and therefore communicate that the number of marbles found by Cody is exactly 11. Obviously, this is a wrong prediction. There have been several proposals to deal with this problem (Krifka 1999; van Rooij and Schulz 2006; Fox and Hackl 2006), but for now it suffices to simply note its existence. We will return to it below. 


\subsection{Issue 2: More than three as a comparative}

The naive approach to modified numerals sketched above assumes that there is no formal link between a modifier like more than and the use of the same and similar expressions in comparative constructions. It turns out, however, that it makes a lot of sense to treat more than three as a regular comparative, rather than as a complex determiner. This has especially been argued by Hackl (2000). One argument he uses will be particularly insightful for what is to follow. The modified numeral fewer than $n$ gives rise to an ambiguity that is also found with regular comparatives. ${ }^{1}$ Consider (6), which is ambiguous between the weak reading in (6a) and a stronger one in (6b).

(6) You are allowed to pick fewer than ten cards.

a. picking 0-9 cards is allowed (compatible with having permission to pick more as well)

b. nine is the highest number of cards you may pick

According to the weak reading, (6) says that a situation where someone picks fewer than 10 cards falls within what is permitted. On this reading, nothing is said about whether one may or may not pick more cards as well. The preferred reading, however, is $(6 \mathrm{~b})$, which gives an upper bound of nine on how many cards may be picked. Exactly the same ambiguity is discussed by Heim (2000) with respect to normal comparatives.

(7) (This paper has 20 pages.) Your paper is allowed to be less long than that.

a. papers of 1-19 pages are allowed (compatible with having permission to write more as well)

b. the longest your paper is allowed to be is 19 pages

Heim accounts for this ambiguity structurally, by proposing that the comparative and the modal can alternate scope. The idea is that the comparative operator plus the than-clause denotes a quantifier over degrees. This quantifier constitutes the degree argument of the adjective and has to quantifier-raise to avoid a type clash. Assuming that comparison involves a maximality operator over degrees, as in (9), the weak and the strong reading fall out automatically. So, (10a) corresponds to the scope ordering in $(8 \mathrm{a})$ and $(10 \mathrm{~b})$ to that in $(8 \mathrm{~b})$.

(8) a. [ allowed [ [less than that $]_{1}$ your paper is $t_{1}$ long ] ]

b. [ [less than that $]_{1}$ [ allowed [ your paper is $t_{1}$ long ] ] ]

(9) $\llbracket$ less than $\alpha \rrbracket=\lambda P\langle d, t\rangle \cdot \max _{d}(P(d))<\alpha$

(10) a. $\diamond\left[\max _{d}(\operatorname{long}(p, d))<20\right]$

b. $\max _{d}(\diamond \operatorname{long}(p, d))<20$

Hackl's observation is now that exactly the same structural ambiguity can be argued to take place with comparative modified numerals, on the assumption that numerals

\footnotetext{
${ }_{1}$ Actually, one can only show that this ambiguity exists for fewer/less than. For reasons explained in Heim (2000), the relevant ambiguities are only visible with non-increasing operators.
} 
involve a covert cardinality operator many. This many can either be interpreted as an adjective (a predicate of cardinalities) or as a cardinality quantifier as illustrated in (12) (see Hackl 2000, Chap. 2, for details).

(11) a. [ allowed [ [fewer than ten $]_{1}$ you pick $t_{1}$ many cards ] ]

b. [ [fewer than ten $]_{1}\left[\right.$ allowed [ you pick $t_{1}$ many cards ] ] ]

$\llbracket$ many $\rrbracket=\lambda d \cdot \lambda P_{\langle e, t\rangle} \cdot \lambda P_{\langle e, t\rangle}^{\prime} \cdot \exists x\left[|x|=d \& P(x) \& P^{\prime}(x)\right]$

$\llbracket$ fewer than ten $\rrbracket=\lambda P_{\langle d, t\rangle} \cdot \max _{d}(P(d))<10$

In parallel to (10), (11a) and (11b) yield the expected readings in (14a) and (14b), respectively.
a. $\diamond\left[\max _{d}(\exists x[|x|=d \& \operatorname{card}(x) \& \operatorname{pick}(y, x)])<10\right]$
b. $\max _{d}(\diamond \exists x[|x|=d \& \operatorname{card}(x) \& \operatorname{pick}(y, x)])<10$

What this shows is that the comparative form of certain modified numerals is to be taken seriously. By just assuming a determiner denotation that relates two cardinalities by $>$ or $<$, one ignores the fact that such modifiers are real derivatives of comparative constructions. One might wonder what this means then for other, noncomparative modifiers of numerals. This is what I turn to next.

\subsection{Issue 3: Distinguishing different modifiers}

According to the naive approach I have sketched, several modified numerals end up

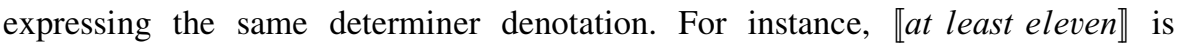

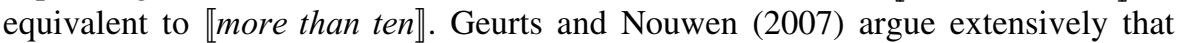
there is no such equivalence. In Sect. 5 I will repeat some of these arguments, but I want to point out here already that superlative modifiers like at most and at least differ from their comparative counterpart with respect to the structural ambiguities discussed in the previous subsection. According to the naive approach, (15) should be equivalent to (6).

(15) You are allowed to pick at most nine cards.

a. \#picking 0-9 cards is allowed

b. ten is the highest number of cards you may pick

Example (15) lacks the weak reading. That is, it is incompatible with a scenario where, say, picking 12 cards is (also) permitted. I will return to this example below. For now it suffices to just keep in mind that formal differences between numeral modifiers are to be taken seriously. ${ }^{2}$ This will become increasingly clear in the next section, where I turn to the semantics of no more than.

\footnotetext{
${ }^{2}$ See Nouwen (2008b), as well as Nouwen (2008a), for more arguments for such a strategy when studying quantifiers.
} 


\section{The semantics of $n o(t)-e r$}

In the introduction, I remarked that if -er is an expression of strict comparison, then its negation will have to be an expression of non-strict comparison. This section tests the linguistic reality of that correspondence.

\subsection{Interpreting no more}

One way to think of no more is to analyse it as being a differential, in parallel to twelve more than or a lot more than. On such a view, we would expect a semantics as in (16) (where $P$ is a property of degrees/cardinalities).

$$
\llbracket \text { no more than } \alpha \rrbracket=\lambda P . \neg \exists d^{\prime}\left[\max _{d}(P(d))=\alpha+d^{\prime}\right]
$$

In Dutch and German, regular negation is used where in English no combines with a comparative.

(17) Sjeng heeft niet meer dan tien knikkers S. has not more than ten marbles gevonden. [Dutch]

found

'Sjeng found no more than ten marbles.'

(18) Werner hat nicht mehr als zehn Murmeln W. has not more than ten marbles gefunden [German]

found

'Werner found no more than ten marbles.'

One would expect the following semantics for the combination of negation and comparison:

$$
\llbracket \text { nicht mehr als } \alpha \rrbracket=\lambda P . \neg\left[\max _{d}(P(d))>\alpha\right]
$$

Note that $\llbracket$ no more than $\alpha \rrbracket$ is equivalent to $\llbracket$ nicht mehr als $\alpha \rrbracket$ and expresses (20): the set of degree properties whose highest degree is not higher than $\alpha^{3}{ }^{3}$ In other words, the semantics of no(t) -er is (20).

$$
\lambda P \cdot \max _{d}(P(d)) \leq \alpha
$$

The above results in the following semantics for (21).

\footnotetext{
${ }^{3}$ (19) holds only for positive adjectives. For negative antonyms the relations simply switch direction. For instance:

(i) $\quad \llbracket$ no fewer than $\alpha \rrbracket=\lambda P . \neg\left[\max _{d}(P(d))<\alpha\right]=\lambda P \cdot \max _{d}(P(d)) \geq \alpha$

Nothing really hinges on the particular implementation, however. One could also adopt an interval based semantics, where negative antonyms operate on inverted scales. See e.g. Kennedy (2001), Heim (2006). 
(21) Cody found no more than ten marbles.

a. [no more than ten $]_{1}$ Cody found $t_{1}$ many marbles

b. $\max _{d}$ (Cody found d-many marbles $) \leq 10$

c. $=$ the total number of marbles found by Cody $\leq 10$

As I will now show, this kind of straightforward approach to the semantics of $n o(t)$ -er fails to yield the correct meanings.

\subsection{Equality readings}

According to the semantics above, no -er constructions express $\leq$ - or $\geq$-relations. In reality, however, such constructions behave as if they were comparatives expressing equality. ${ }^{4}$ This already becomes clear from such simple examples as (21). Its preferred interpretation is that Cody found exactly ten marbles. Moreover, it has an evaluative side-effect of expressing that ten marbles does not count as a lot. Similarly, (22) says that fifty people showed up and that this can be considered to be many.

(22) (The organisers expected a small audience. However,) no fewer than fifty people showed up.

I am not the first person to notice such data. ${ }^{5}$ Jespersen (1966), for instance, remarks that "no less than 30 means exactly 30, implying surprise or wonder at the high number" (p. 83). Elsewhere (Jespersen 1949, entry 16.842 on p. 434), he equates no more than to as little as and explores the full range of uses of the no more construction (entries 16.83-16.86). Jespersen notes that there is a difference between no and not in combinations with comparatives. This contrast, he notes, had already been observed by Stoffel (1894), who for instance discussed the quote "The victorious emperor remained at Rome not more than three months." Stoffel comments on it in the following way: "This means that he remained three months at most; if the author had written 'no more than three months', this form of expression would have implied that the author thought this a brief period, and 'no more than three months' would be equivalent to 'three months only'" (Jespersen 1949, p. 435).

I will ignore the difference between no more and not more for a while and return to it later. For now, let it be clear that the general observation is that no more does not express an upper bound, but rather an equality. Similar observations can be made outside the domain of modified numerals. For instance, the so-called whale construction (cf. Sawada 2005) uses no more/fewer as an equality.

(23) A whale is no more a fish than a horse.

\footnotetext{
4 In an earlier version of this article, I used the terminology equative to describe the double-bounded interpretation of no $(t)$-er constructions. As rightly pointed out by an anonymous reviewer, the term equative is misleading, as equative constructions are usually assumed to express the $\geq$-relation and not the =-relation (von Stechow 1984). By an equality reading, I mean a reading involving the latter relation.

5 I'm indebted to Doris Penka for pointing out relevant passages in Otto Jespersen's work.
} 
What (23) says is that the extent to which a whale is a fish equals the extent to which a horse is a fish. Similarly, (24) does not say that Holland's size is at mòst that of a very big city, but rather equates it to very-big-city size.

(24) a. Holland is no more than a very big city.

b. Nederland is niet meer dan een zeer grote stad the Netherlands is not more than a very big city 'You could compare the Netherlands to a very big city.

The conclusion is that no -er constructions are generally interpreted as equalities.

\subsection{Towards an explanation}

There is a straightforward way to connect the desired interpretation of (25), in (25a), to the interpretation (25b) which is derived using the semantics we expect from the interaction of negation and comparison: (25a) is the exhaustified interpretation of (25b). The reading in (25b) is asymmetrically entailed by the alternatives in (26). The reading in (25a) is equivalent to the conjunction of (25b) and the denial of the alternatives in (26).

(25) No more than thirty people showed up.

a. $\max _{d}(d$-many people showed up $)=30$

b. $\max _{d}(d$-many people showed up $) \leq 30$

$$
\max _{d}(d \text {-many people showed up }) \leq 29
$$

$\Leftarrow \max _{d}(d$-many people showed up $) \leq 28$

$\Leftarrow \max _{d}(d$-many people showed up $) \leq 27$

$\Leftarrow \max _{d}(d$-many people showed up $) \leq 26$

In other words, the simple non-strict comparison semantics for no more gives rise to the correct interpretation once we assume that it is exhaustified in a standard way (by denying stronger alternatives). This is surprising. As explained in Sect. 2, comparative modified numerals generally lack implicatures. However, the above suggests that this only holds for strict comparison. The question is, now, why there should be a difference between strict and non-strict comparison with respect to exhaustification.

Before I turn to that question, I will discuss some more complicated data, concerning scopal interactions, which corroborate the point made so far in this section.

\subsection{Scope alternation with modals}

The equality readings of negative comparison are strikingly illustrated when we turn to modal sentences. Consider (27).

(27) Cody's paper is allowed to have no more than 20 pages.

As I discussed above, comparatives give rise to scope ambiguities when embedded under a modal operator. Given a non-exhaustive semantics for 'no -er', we would then expect the following two readings for (27). 
(28) a. $\diamond\left[\max _{d}\right.$ (Cody's paper has $d$-many pages $\left.) \leq 20 \mathrm{pp}\right]$

b. $\max _{d}(\diamond[$ Cody's paper has $d$-many pages $]) \leq 20 \mathrm{pp}$

One of the possible readings of (27) is (28a). However, it is only available in echoic uses and needs a distinctive stress pattern. For example, imagine somebody thinking Cody's paper is too short, that it should be longer than 20 pages. This can be contested by saying: You're wrong, Cody's paper IS allowed to have no more than 20 pages.

The reading in (28a) is certainly not the preferred reading for (27). What about (28b)? This says that papers with 21 or more pages are not acceptable. Notice that it does not indicate where exactly the page limit is. Specifically, (28b) does not entail that 20-page papers are allowed. This is a wrong prediction, which can be illustrated by the contrast in (30).

(29) Cody's paper is allowed to have no more than 20 pages.

$\Rightarrow$ Cody's paper is allowed to have 20 pages.

(30) a. Cody's paper is allowed to be have less than 20 pages. In fact, the page limit is 15 pages.

b. Cody's paper is allowed to be have no more than 20 pages. \#In fact, the page limit is 15 pages.

An example like (27) tells the hearer exactly what the upper limit on the number of pages is. The preferred reading for (27) is (31).

$\max _{d}(\diamond[$ Cody's paper has $d$-many pages $])=20 \mathrm{pp}$

This is the same observation as I made before: on the basis of the semantics I proposed for negative comparatives, we expect to find an interpretation involving non-strict comparison, but in fact a strong equality reading is found.

Similar data exist with universal modals as well. Comparatives embedded under require yield lower-bounded readings when the maximality operator in the semantics of -er scopes over the modal. For instance, (32) has a reading which says that the smallest amount of publications that would still make Cody eligible for tenure is smaller than four. In other words, there is a number smaller than four such that Cody needs to publish at least that number of papers. (See Heim 2000 and Hackl 2000 for details. These examples and the context that helps to trigger the relevant scope ordering are based on examples in Hackl's work.)

(32) Cody is not eligible for tenure at MIT. He didn't publish enough articles.

He can try to get tenure at Acme University, however. There, one is required to have published fewer than four papers.

The same effect can be observed with no -er comparatives under require, with one difference. Unlike (32), in (33) the resulting reading specifies an exact lower bound on how many papers Cody needs to publish. That is, (33) says that this number is 
three. In other words, (33) entails that for tenure at Acme, Cody needs to publish three articles. Such an entailment is absent in (32).

(33) Cody is not eligible for tenure at MIT. He didn't publish enough articles. He can try to get tenure at Acme University, however. There, one is required to have published no more than three articles in order to get tenure.

Above, I showed that the equality readings can be derived from the readings generated by the non-strict comparison semantics for no -er by means of exhaustification; the same applies to the examples in the current section. For instance, (34a) is asymmetrically entailed by the alternatives in (34b), and, so, (34c) can be seen as the exhaustification of (34a).

(34) Cody's paper is allowed to have no more than 20 pages.

a. $\max _{d}(\diamond[$ Cody's paper has $d$-many-papers $]) \leq 20$ pp.

b. $\Leftarrow \max _{d}(\diamond[$ Cody's paper has $d$-many papers $]) \leq 19$ pp.

$\Leftarrow \max _{d}(\diamond[$ Cody's paper has $d$-many papers $]) \leq 18$ pp.

$\Leftarrow \max _{d}(\diamond[$ Cody's paper has $d$-many papers $]) \leq 17 \mathrm{pp}$.

c. $\max _{d}(\diamond[$ Cody's paper has $d$-many papers $])=20 \mathrm{pp}$.

Notice that we do not expect an equality reading when the modal takes widest scope, as in (28a), repeated in (35), since such a reading does not line up on an entailment scale like the one in (34b).

$$
\left.\diamond\left[\max _{d} \text { (Cody's paper has } d \text {-many pages }\right) \leq 20 \mathrm{pp}\right]
$$

As mentioned above, an analysis of the equality readings that uses exhaustification is puzzling, for it begs the question why some modified numerals should be subject to exhaustifications, while others are not. I now turn to this issue.

\section{Exhaustification and comparison}

What I suggested in the last section is that the equality readings for no -er comparatives are the result of a straightforward semantics for negation and comparison, coupled with exhaustification. In other words, I claim that there is nothing mysterious about the semantics of such constructions. The problem is rather how to account for the possibility of generating implicatures. In order to solve this problem, I return to the discussion of implicatures for modified numerals that I briefly touched upon in Sect. 2. As it turns out, one specific proposal for the lack of implicatures for more than numerals, namely Fox and Hackl (2006), predicts a contrast between strict and non-strict comparison.

\subsection{The universal density of measurements}

The original puzzle was why if Cody found more than ten marbles, there is no implicature denying he found more than eleven. Fox and Hackl (2006) propose a 
radical solution to this puzzle, couched in a principle that states that natural language semantics operates with only one kind of scale.

\section{The Universal Density of Measurements (UDM):}

Measurement scales that are needed for natural language semantics are always dense.

(Fox and Hackl 2006, p. 542)

Given the UDM, there is an elegant explanation of why more than ten does not imply not more than eleven. Say that (37a) has the semantics in (37b).
a. Cody found more than ten marbles.
b. $\max _{d}$ (Cody found $d$-many marbles) $>10$

Given (37b), we are interested in what the stronger alternatives are. Standard accounts would now say that they are of the form ' $\max _{d}$ (Cody found $d$-many marbles) $>n$ ', with $n$ a natural number exceeding 10. However, given the UDM, we are forced to consider other stronger alternatives as well. In fact, there now is a dense (open) scale $(10, \infty)$ which yields the asymmetrically entailing alternatives. The implicatures are:

$$
\neg\left[\max _{d} \text { (Cody found } d \text {-many marbles) }>n\right] \quad \text { with } n \in(10, \infty)
$$

These implicatures, however, end up contradicting the assertion. Here's why: (37b) asserts that there is a $d$ such that Cody found $10+d$ marbles. Whatever $d$ we try, there will be an implicature denying that Cody found more than $10+d / 2$ marbles. The implicatures, therefore, will have to be rejected.

A different prediction is made once we turn to non-strict comparison. A meaning as in (39a) generates the implicatures in (39b).
a. $\max _{d}$ (Cody found $d$-many marbles) $\geq 10$
b. $\neg\left[\max _{d}\right.$ (Cody found $d$-many marbles) $\left.\geq n\right]$
with $n \in(10, \infty)$

(39a) says that there is a $d \in[0, \infty)$ such that Cody found $10+d$ marbles. For each $d>0$, there is an implicature denying that Cody found $10+d / 2$ marbles. No implicature denies the possibility of Cody finding $10+0$ marbles. Consequently, if we accept the implicatures, we arrive at Cody finding exactly ten marbles. The UDM predicts, then, that any natural language sentence that expresses (39a) will be interpreted exhaustively. More generally, the predictions are as follows:

(40) UDM predictions:

* Strict comparison does not yield sensible implicatures

* Non-strict comparison does yield sensible implicatures

These predictions account for the data I discussed in Sect. 3. Any meaning of the form $d \leq d^{\prime}$ or $d \geq d^{\prime}$ will be exhaustified to $d=d^{\prime}$. For instance: 
(41) No more than thirty people showed up.

a. $\max _{d}(d$-many people showed up $) \leq 30$

b. implicatures: $\neg\left[\max _{d}(d\right.$-many people showed up $\left.) \leq \mathrm{n}\right]$ with $n \in[0,30)$

c. (a.), (b.) $\Rightarrow \max _{d}(d$-many people showed up) $=30$

Exactly the same reasoning holds for (42a), when interpreted as (42b).

(42) a. Cody's paper is allowed to have no more than twenty pages.

b. $\max _{d}(\diamond[$ Cody's paper has $d$-many pages $]) \leq 20$ pp

The implicatures say that there is no $n<20$ such that the upper limit on Cody's paper is smaller or equal to $n$. Taking these implicatures into account, there is only one possibility left for what the upper limit is: Cody's paper can't be longer than 20 pages.

\section{More modified numerals}

The UDM's predictions are very sharp and although they suit the data for no more very well, it needs to be checked whether they do not over-generate exhaustive readings. Apart from no -er constructions, there are other candidates for the expression of non-strict comparison. All of these should now yield implicatures. With respect to (43), however, this seems to be an unwanted prediction.

(43) Cody found at least ten marbles.

is not understood as: Cody found exactly ten marbles

The claim I want to make about such examples is that it is a mistake to think of the constructions involved as cases of non-strict comparison.

\subsection{Superlative modified numerals}

Consider the word height.

(44) There is more than one ' $\mathrm{h}$ ' in height.

Someone uttering (44) is speaking the truth, at least as far as English spelling rules are concerned. Something different happens if we substitute the comparative modified numeral with what the naive account from Sect. 2 considered its superlative equivalent.

(45) There are at least two 'h's in height.

From (45) we normally conclude that the speaker does not know how to spell height. (S/he is, for instance, unsure whether it is 'height' or 'heighth' - the latter perhaps in analogy to 'width'). Similarly:

(46) There are at most two 'h's in height. 
Again, (46) is normally said by someone who does not know how to spell. But why would that be? If (45) and (46) express non-strict comparison, then they should correspond to (47a) and (47b) respectively. With regard to English spelling rules, both of these express a truth.
a. the number of ' $h$ 's in height $\geq 2$
b. the number of 'h's in height $\leq 2$

Notice that one cannot argue that, although true, (47a) and (47b) are simply too weak to express how many letters ' $h$ ' there are in height. For if that reasoning were to hold, it should also apply to (44), which expresses the true but weak statement that the number of ' $h$ 's in height $>1$.

So why do the at least/most examples imply the speaker's ignorance about English spelling? Geurts and Nouwen (2007) explain this by arguing that the semantics of superlative modifiers express modal attitudes to alternatives on a scale. So, rather than to (47) the examples in (45) and (46) correspond to the following.

(48) There are at least two 'h's in height.

$\square[$ height has two ' $h$ 's] \& $\diamond$ [height has more than two 'h's]

(49) There are at most two 'h's in height.

$\diamond[$ height has two 'h's] \& $\neg \diamond[$ height has more than two 'h's]

By default, the modality expressed by superlative modifiers is epistemic. Thus, (48) explicitly states that the speaker considers it possible that the word height has more than two occurrences of the letter ' $h$ '. If one knows about the spelling of height, this would render an utterance of (48) false. Similarly, (49) leaves open the possibility that height is spelled with just one ' $h$ ' (like 'hite').

There is considerable support for the semantics in (48) and (49). See Geurts and Nouwen (2007) for detailed theoretical argumentation and Geurts (2007) and Geurts et al. (2008, unpublished manuscript) for experiments confirming the theory's predictions. To mention just one argument for the semantics above, recall from Sect. 2 that in contrast to comparative numeral modifiers, superlative modifiers do not trigger an ambiguity when embedded under a modal. So, (50) can only mean that the upper bound on how many cards one is allowed to pick is 10. It lacks a weaker reading.

You are allowed to pick at most ten cards.

This is predicted by Geurts and Nouwen since the modal semantics for at most explicitly denies the possibility of higher alternatives. Moreover, epistemic modality generally takes widest scope, so scope alternations are not to be expected with 'allowed'.

The solution to why at least/most do not yield implicatures follows from similar reasoning. The semantics of superlative modifiers is comparable to that of only or to the exhaustivity operator. Superlative modifiers use up the alternatives in their semantics. 


\subsection{Other numerals}

Superlative numeral modifiers are not the only remaining candidates for the expression of non-strict comparison. The observation is, however, that the no more/ fewer construction is the only one which results in equality readings.

(51) Cody found at most/least ten marbles. is not understood as: Cody found exactly ten marbles

(52) Cody found ten or fewer/more marbles. is not understood as: Cody found exactly ten marbles

(53) Cody found up to ten marbles.

is not understood as: Cody found exactly ten marbles

The explanation of why such constructions lack implicatures is simple, however. They just do not express (non-strict) comparison: the direct object in (51) is a superlative construction, the one in (52) is a disjunction, and the one in (53) is a PP. These come with their own semantics, which might resemble non-strict comparison in certain ways, yet at the same time (as became clear from the discussion of at least/most) differ from comparison in subtle but crucial ways.

The case of up to is comparable to that of superlative modifiers. Up to is a complex directional preposition with an extremely limited distribution. ${ }^{6}$ In the domain of numerals, it is similar to at most. For instance, when embedded under a modal, it resists weak readings.

(54) Cody is allowed to pick up to four cards.

Parallel to the at most data, (54) expresses an upper bound on what Cody is allowed to pick. It cannot express anything weaker. Like at most, it seems as if up to consumes alternatives to express an upper bound. This becomes clear from (55), which (if acceptable) can only be interpreted as the speaker allowing some variability in the spelling of height. It cannot be interpreted as a simple yet weak truth spoken by a competent speller.

There are up to two 'h's in height

With respect to the observation in (52), a rather different kind of explanation can be found in Fox (2007). Crucially, however, Fox's explanation rests on $n$ or more conforming to the principles that govern the semantics of disjunction. I will not go into Fox's reasoning here, but return to it in Sect. 7.

\section{Interim conclusion and tying some loose ends}

The plot has been as follows. I have shown that no -er comparatives express equality, and argued that on a straightforward semantics for negation and

\footnotetext{
${ }^{6}$ See Nouwen (2008a) for a detailed account.
} 
comparison this can be explained if these constructions yield implicatures. Since strict comparatives are never exhaustified, this begs the question why there should be such a difference. Fox and Hackl's principle of the universal density of measurements, however, makes exactly this prediction.

The foregoing contributes to the increasing body of literature that argues for a more fine-grained view on the varied class of modified numerals that languages like English have. (See Nouwen (2008b) for a programmatic statement.) What is crucial for this paper is that there are three kinds of modified numerals:

1. numeral modifiers expressing $<$ or $>$ - these resist exhaustification example: more than

2. numeral modifiers expressing $\leq$ or $\geq$-these yield implicatures example: no more than

3. other modifiers, which do not straightforwardly express a comparison relation example: at least

So far, I have presented a compositional semantics of no -er constructions, with particular attention to how these constructions relate to comparison relations. I have not, however, explained the full meaning potential of such comparatives. In the remainder of this section, I will briefly touch upon two loose ends.

\subsection{Evaluative readings}

The equality reading of no -er constructions comes with an evaluative flavour.

a. no fewer than ten marbles flavour: that's a lot

b. no more than ten marbles flavour: that's not a lot

In general, adjectives in a marked form (e.g. the positive forms of negative adjectives, equatives of negative adjectives) yield evaluative readings (cf. for instance Bierwisch 1989). If Cody is as tall as Vic, nothing follows about Cody's and Vic's height except that these are the same. However, if Cody is as short as Vic, it is suggested that Cody and Vic are both short. Whatever the precise explanation of this phenomenon is, part of it will have to be the fact that the marked form as short as is expected to yield a marked interpretation. If someone simply wants to convey that Cody and Vic have the same height, then using an equative with the positive adjective is the most unmarked way of doing so. Evaluative readings emerge as a side effect of marked forms.

A similar reason could account for the evaluative flavours of (56). The unmarked way of referring to a group of 10 marbles is just saying ten marbles. Any more involved way of reaching the same effect is bound to have an evaluative side effect. For instance, as many/few as is equally evaluative. ${ }^{7}$
a. as many as ten marbles flavour: that's a lot
b. as few as ten marbles flavour: that's not a lot

\footnotetext{
${ }^{7}$ I am grateful to Irene Heim for bringing this to my attention.
} 
I will refrain from trying to go into more detail about how evaluative readings are derived. ${ }^{8}$ The main point is this: evaluativity is to be expected for no -er constructions, given that such constructions would otherwise be rather marked forms that come with very unmarked interpretations.

\subsection{Negation}

There is a clear difference between (58a) and (58b):

(58) a. You are allowed to pick no more than ten cards.

b. You are not allowed to pick more than ten cards.

c. $\neg \diamond\left[\max _{d}(\ldots)\right]$

As observed, the preferred reading of (58a) says that 10 cards is the upper bound on what one is allowed to pick. In contrast, (58b) says something weaker: picking more than ten cards is not allowed. In other words, $(58 \mathrm{~b})$ has the normal non-exhaustified meaning in $(58 \mathrm{c}){ }^{9}$

The same contrast is found in non-embedded comparatives. In general, no -er has an equality semantics, whereas not -er has a non-strict comparative one. (As mentioned above, this contrast was already noticed by Jespersen and Stoffel.)

(59) a. Cody is not more athletic than Vic.

b. Cody is no more athletic than Vic.

Sentence (59b) strongly suggests that neither Cody nor Vic are athletic. In contast, (59a) simply denies that Cody is more athletic than Vic. That is, (59a) has no double-bounded meaning, nor does it yield an evaluative flavour.

These data, however, are to a certain extent questionable. I believe both that not -er can have an (evaluative) equality meaning, and that no -er can have a non-strict comparison (upper- or lower-bounded only) meaning. An example of the latter is (60). ${ }^{10}$

(60) According to EU law, passenger cars are allowed to be $2.50 \mathrm{~m}$ wide, but no wider.

Clearly, no wider has its unstrengthened strict comparison sense here, since a parallel example with an explicit equative is unacceptable:

(61) \#According to EU law, passenger cars are allowed to be $2.50 \mathrm{~m}$ wide, but/and as wide as that.

It seems that the negation involved in no(t) -er constructions can either be simple (sentential) negation or be interpreted as a denial, where I take denial to involve widest scope negation. Since exhaustification is only available with non-strict comparison, we now get the following options for a no(t) -er construction. When

\footnotetext{
${ }^{8}$ See Rett (2007) for a detailed formal approach to evaluativity.

${ }^{9}$ Note that $(58 \mathrm{c})$ is equivalent to (i), the non-exhausted reading for (58a):

(i) $\neg\left[\max _{d}(\diamond[\ldots])>10\right]$

10 Jespersen (1949, entry 16.86, p. 437) hints at a similar example.
} 
negation is construed with narrow scope, exhaustification can take place since the negation and the semantics of -er result in non-strict comparison. The result is the expression of equality. Alternatively, no exhaustification takes place and the result is simply non-strict comparison. If the negation is taken to be a denial, exhaustification cannot occur, for it would have to apply to strict comparison. Consequently, when no $(t)$-er involves denial, it will have to be interpreted as the denial of strict comparison. Schematically:

(62) No negation/denial:

$[>]=$ regular strict comparison

$\operatorname{exh}[>]=$ not available

(63) Negation:

$[\neg>]=$ non-strict comparison

$\operatorname{exh}[\neg>]=$ equality

(64) Denial:

Denial of $[>]=$ non-strict comparison

Denial of $\operatorname{exh}[>]=$ not available

The data above suggest that the role of denial is preferably played by not, whereas no is non-widest scope negation in the semantics. In Dutch and German, where the negated comparison construction is always expressed with regular negation, there is general ambiguity.

(65) Sjeng heeft niet meer dan tien knikkers gevonden.

S. has not more than ten marbles found

The Dutch example (65) either means that Sjeng found a meager ten marbles, or that he didn't find more than 10 . The latter reading, however, is only available with stress on the auxiliary. ${ }^{11}$

\footnotetext{
${ }^{11}$ An anonymous reviewer wonders why English would allow negative determiners to express the nonexistence of a difference degree, while Dutch and German do not, and observes that English has related constructions which do not involve cardinality, such as e.g. no longer, which also seem to be lacking in other languages. The reviewer moreover expresses the worry that such non-cardinality-based constructions do not yield equality readings, given examples like (i) (from Google).
}

(i) One study concluded that, on average, middle-aged female smokers live no longer than male smokers.

I share the reviewer's intuition concerning (i), but do not think this example is representative. As mentioned above, it should come as no surprise that (evaluative) equality readings are sometimes lacking from no -er constructions. I believe that, in general, negative comparatives not involving cardinality behave exactly like those that do. This, in any case, was the intuition already of Stoffel (1894), who notices the contrast between (iia) and (iib).

(ii) a. He isn't more to be trusted than you are

b. He is no more to be trusted than you are

Stoffel proposes to paraphrase (iia) as 'I deny that he is more to be trusted than you are' (p. 88), and then states that "in 'he is no more to be trusted than you are', we have the [...] negative no prefixed to a comparative, and here it has the force of changing the sense of more than into that of as little as" (ibid). 
In sum, it is expected that there is some variability in the readings no $(t)$-er gives rise to, given the optionality of exhaustivity as well as the option of interpreting the negation as a form of denial. In Dutch and German this results in a genuine ambiguity for such constructions. In English, this ambiguity is less clearly present. I suggested that this may be due to there being two constructions no -er versus not -er, the latter being more suitable to express denial.

\section{Discussion}

One might wonder whether it is possible to obtain an account of the no(t) -er data that is similar to the one above, but does not involve assuming the UDM. In this final section, I discuss some salient alternatives.

\subsection{Dynamic exhaustivity}

One option (suggested to me by Robert van Rooij) is to blame the difference in exhaustification between more than and no more than on the occurrence of negation in the latter. Before I can investigate this option, I first need to discuss an alternative explanation of why simple comparative modified numerals do not yield implicatures.

In van Rooij and Schulz (2006), exhaustification is proposed to be interpretation in minimal models. Roughly, the proposal is as follows. Exhaustification occurs with respect to a background question predicate. Such a predicate defines an order on possible worlds: $w$ is more minimal than $w^{\prime}$ with respect to $P$, or $w<_{P} w^{\prime}$, if and only if the extension of $P$ in $w$ is a proper subset of the extension of $P$ in $w^{\prime}$. The exhaustive interpretation of $\varphi$ w.r.t. $P$ is now the set of worlds in $\varphi$ that are lowest on the $<_{P}$-scale. For instance, (66), when interpreted as (66a) against the background question in (66b), gets the exhaustive interpretation in (66c). The reader may check that this is the set of worlds in which Cody found exactly three marbles.

(66) Cody found three marbles.

a. $\xi=\exists x[\operatorname{marble}(x) \&$ found $(c, x) \&|x|=3]$

b. $P=\lambda x \cdot \operatorname{marble}(x) \&$ found $(c, x)$

c. $\operatorname{exh}(\xi, P)=\left\{w \in \xi \mid \forall w^{\prime} \in \xi: w \neq w^{\prime} \rightarrow w<{ }_{P} w^{\prime}\right\}$

With a small modification, the minimal models approach to exhaustification can also account for why a modified numeral like more than three fails to implicate not more than four. According to van Rooij and Schulz, all one needs to assume is that (minimal) models do not only involve possible worlds, but moreover involve assignment functions. In other words, the models taken into account are those of dynamic, rather than static, semantics. So, say that $i$ is a possibility $\langle w, f\rangle$, a possible world paired with an assignment $f$, and that likewise $i^{\prime}=\left\langle w^{\prime}, f^{\prime}\right\rangle$. Now, $i \ll_{P} i^{\prime}$ if and only if $f=f^{\prime}$ and $w<_{P} w^{\prime}$.

This changes nothing for (66). In case there are four marbles, $m_{1}, m_{2}, m_{3}$ and $m_{4}$, the possibilities that are left after update with $\xi$ contain worlds in which Cody found 
three or more marbles, paired with assignment functions that map any trio of found marbles to $x$. Say now $w^{\prime}$ is a world in which Cody picked all four marbles. There are four possibilities that contain this world following an update with $\xi$, and they are: $\left\langle w^{\prime}, x:=m_{1} \oplus m_{2} \oplus m_{3}\right\rangle,\left\langle w^{\prime}, x:=m_{1} \oplus m_{3} \oplus m_{4}\right\rangle,\left\langle w^{\prime}, x:=m_{1} \oplus m_{2} \oplus m_{4}\right\rangle$, and $\left\langle w^{\prime}, x:=m_{2} \oplus m_{3} \oplus m_{4}\right\rangle$. None of these possibilities is minimal with respect to $P$, for there will exist a world in which Cody picked exactly three marbles that is paired with one of the assignment functions $w^{\prime}$ is paired with. For instance, if $w$ is a world in which $m_{1}, m_{2}$, and $m_{3}$ were found, then $\left\langle w, x:=m_{1} \oplus m_{2} \oplus m_{3}\right\rangle \leftarrow_{P}\left\langle w^{\prime}, x:=m_{1}\right.$ $\left.\oplus m_{2} \oplus m_{3}\right\rangle$. The same holds for the other possibilities containing $w^{\prime}$ : the most minimal possibilities will only contain worlds like $w$. Consequently, the exhaustive interpretation is one where Cody found exactly three marbles.

Things change when we turn to modified numerals. ${ }^{12}$

Cody found more than two marbles.

a. $\varphi=\exists x[x=\Sigma y[\operatorname{marble}(y) \&$ found $(c, y)] \&|x|>2]$

b. $P=\lambda x \cdot \operatorname{marble}(x) \&$ found $(c, x)$

c. $\operatorname{exh}_{\text {(dynamic) }}(\varphi, P)$ w.r.t. context $j=$ $\left\{\langle w, f\rangle \in[\varphi] j \mid \forall\left\langle w^{\prime}, f^{\prime}\right\rangle \in[\varphi] j:\left(w \neq w^{\prime} \& f=f^{\prime}\right) \rightarrow w<_{P} w^{\prime}\right\}$

Assume once more that $w$ is a world in which Cody found three marbles and $w^{\prime}$ one in which he found four. In an empty context $j$ :

$$
\begin{aligned}
& \text { a. }\left\langle w, x:=m_{1} \oplus m_{2} \oplus m_{3}\right\rangle \in[\varphi] j \\
& \text { b. }\left\langle w^{\prime}, x:=m_{1} \oplus m_{2} \oplus m_{3} \oplus m_{4}\right\rangle \in[\varphi] j
\end{aligned}
$$

The possibility in (68b) is just as minimal as the one in (68a). This is because $i \ll_{P} i^{\prime}$ only in case $i$ and $i^{\prime}$ come with the same assignment function. So even though $w<_{P} w^{\prime}$, the two possibilities in (68) do not stand in the dynamic $\lessdot_{P}$-relation because their assignment functions differ. The result is that the exhaustive interpretation will contain worlds like $w^{\prime}$ where Cody found more than three marbles. Thus, van Rooij and Schulz are able to account for the puzzle of why more than two and three differ in exhaustification while at the same time they are equivalent. The insight is that the equivalence only holds in static logic, but disappears when dynamics is taken into account.

\subsection{Dynamics versus density}

Note that van Rooij and Schulz predict no difference between strict and non-strict comparison. The possibilities left after an update with $\psi$ in (69) will be exactly the same as those following $\varphi$ in (67a). That is, $\varphi$ and $\psi$ are dynamically equivalent.

\footnotetext{
${ }^{12}$ I use $\Sigma$ here as the abstraction operator, as in Kamp and Reyle (1993). $\Sigma y[\varphi]$ returns the sum of all individuals $y$ that verify $\varphi$. The use of abstraction is not essential to van Rooij and Schulz's dynamic account of why modified numerals resist exhaustification. The use of abstraction is essential, however, with downward entailing modified numerals in order to escape the so-called 'van Benthem trap' (van Benthem 1986): non-positive quantifiers containing existential quantification are in danger of having trivial or overly weak truth conditions.
} 


$$
\psi=\exists x[x=\Sigma y[\operatorname{marble}(y) \& \text { found }(c, y)] \&|x| \geq 3]
$$

The question is, however, to what extent (69) corresponds to Cody found no fewer than three marbles. Van Rooij (personal communication) suggests to take the negation in no -er constructions seriously to yield (70).

$$
\psi^{\prime}=\neg \exists x[x=\Sigma y[\operatorname{marble}(y) \& \text { found }(c, y)] \&|x|<3]
$$

In dynamic semantics, negation is interpreted as a test. In any context, $\psi^{\prime}$ will fail to change the value for $x$. Consequently, there is no role for the variable assignments in dynamic exhaustification. The result is that the exhaustive interpretation of $\psi^{\prime}$ is fully determined by selecting the most $P$-minimal worlds in which Cody did not find fewer than three marbles. These are the worlds in which Cody found exactly three marbles.

Actually, things are a little bit more complex than this. Note that it would be wrong to predict now that the same reasoning applies to no more than, for the most minimal worlds in which Cody did not find more than three marbles are the worlds in which he didn't find any. One needs to assume that negation flips the scale and that, suddenly, we are interested in the most maximal worlds. This is needed anyway to prevent Cody didn't find all marbles from being interpreted as Cody not finding any marbles. For $\psi^{\prime}$ in (70), one needs to assume then that there is a double flipping, one by the negation, one by the $<$. Consequently, for (70), it is minimal worlds that count once again.

If van Rooij's suggestion is on the right track, then there is no longer any reason to believe in a difference between the implicatures of strict and those of non-strict comparison. The only reason we observe a difference is that non-strict comparison is expressed using negation, whereas strict comparison is expressed by a lexical primitive.

But how do we choose between $\psi$ and $\psi^{\prime}$ ? In static logic the two are equivalent. The only difference is their dynamics. How do we choose which is the best dynamic profile? Normally, we would look at anaphora. As far as no more/fewer than is concerned, however, a representation as $\psi$ seems preferable over $\psi^{\prime}$, for such modified numerals do not seem to block pronominal reference in discourse. An analysis as in (71b) would therefore be problematic: the last occurrence of $x$ is dynamically bound in (71a), but not in (71b).

(71) Cody found no fewer than three marbles. He keeps them in a little box.

a. $\exists x[x=\Sigma y[\operatorname{marble}(y) \&$ found $(c, y)] \&|x| \geq 3] \&$ keeps_in_box $(c, x)$

b. $\neg \exists x[x=\Sigma y[\operatorname{marble}(y)$ \& found $(c, y)] \&|x|<3]$ \& keeps_in_box $(c, x)$

The reality is that we found two sentences which are truth-conditionally equivalent, have the same potential for subsequent pronominal anaphora, yet differ in their exhaustive interpretation. That is, the motivation for choosing the static representation $\psi^{\prime}$ over the dynamic $\psi$ would be motivated by exhaustivity only. ${ }^{13}$

\footnotetext{
${ }^{13}$ Note, however, that one could argue that tests like (71) are not suitable to determine the dynamics of the underlying semantic representation, given the existence of techniques in dynamic semantics to account for anaphora that involve essentially static interpretations. See, for instance, the treatment of generalised quantifiers in Kamp and Reyle (1993). See Nouwen (2007) for discussion.
} 
In other words, one would have to disconnect anaphora and dynamics for this to work.

There is another way in which van Rooij and Schulz's dynamic account is separated from a theory of discourse. In some specific contexts, dynamic exhaustivity will give the wrong result if the context's actual information state, inclusive its assignment to variables, is taken into account. Take the following example. Say we are talking about four friends of ours: Ann, Bob, Carl, and Dirk. All four of them have taken an exam last week. You know that Ann, Bob, and Carl passed, but you have had no information as yet about Dirk. Then you hear the following:

Three of your friends passed the exam.

According to the interpretation $\exists x[\operatorname{friend}(x) \& \operatorname{pass}(x) \&|x|=3]$, there are now two possibilities left. Either the actual world is such that exactly three of your friends passed, call this world $w$, or the world is such that all four of your friends passed, call this world $w^{\prime} .{ }^{14}$ In the context described above, there is only one possible assignment function that can be paired with a world, like $w$, in which exactly three friends passed. That is, if exactly three friends pass, then we are in $w$ and $x$ must be assigned the group made of Ann, Bob, and Carl. There will be no possibility that pairs a world other than $w^{\prime}$ with a function assigning Dirk as part of the value for $x$. To be precise, the possibilities after updating with (72) are:

$$
\begin{aligned}
& i_{1}=\langle w, x:=a \oplus b \oplus c\rangle \\
& i_{2}=\left\langle w^{\prime}, x:=a \oplus b \oplus c\right\rangle \\
& i_{3}=\left\langle w^{\prime}, x:=a \oplus b \oplus d\right\rangle \\
& i_{4}=\left\langle w^{\prime}, x:=a \oplus c \oplus d\right\rangle \\
& i_{5}=\left\langle w^{\prime}, x:=b \oplus c \oplus d\right\rangle
\end{aligned}
$$

Dynamic exhaustification will exclude $i_{2}$ (since $i_{1}$ is more minimal), but no other possibility (since the assignments differ). In other words, in this particular context one would expect that exhaustification does not deliver an exactly reading for the bare numeral. Such context dependence of implicatures is not observed, however. Van Rooij and Schulz can escape these predictions by assuming that, as far as exhaustification is concerned, all possibilities should always be taken into account. That is, the process of deriving an exhaustive interpretation operates on the full update potential of expressions, not on their effect in the actual context. In this respect there is a striking similarity between Fox and Hackl's density thesis and van Rooij and Schulz's dynamic semantic proposal. Both accounts rely on the assumption that exhaustive interpretation takes place on a level that is independent from contextual interpretation. Just like the UDM claims that possibilities we would never consider (as, for instance, Cody having three and a half children) have a pragmatic effect, van Rooij and Schulz have to assume that the knowledge we have gained about the discourse is ignored as far as exhaustification is concerned.

\footnotetext{
${ }^{14}$ I'm simplifying here. There would be many more worlds, but for the purpose of these examples they would be exactly like $w$ or $w^{\prime}$ with respect to the relevant properties.
} 
Although this observation might be somewhat surprising, I can see no theoretical objections against either van Rooij and Schulz's or Fox and Hackl's assumption.

There is, however, another complication with van Rooij's suggestion that the negation in no -er constructions rather than density is to blame for the observed implicatures. Note that the predicate logical forms above are rather classic representations of a sentence containing a modified numeral. Such representations are unsuitable for capturing the observation of Hackl (2000) that comparatively modified numerals display the same ambiguities as ordinary comparatives when embedded under an intensional/modal operator (see Sect. 2.2). For instance, (74a) has a reading in which six is the upper bound on how many cards Cody is allowed to pick. But if we assume that its interpretation should be based on (74b) then there is a problem. There is no position in (74b) where inserting a weak modal operator would yield the intended meaning of (74a).

(74) a. Cody is allowed to pick fewer than six cards.

b. $\exists x[x=\Sigma y[\operatorname{card}(y) \& \operatorname{pick}(c, y)] \&|x|<6]$

In order to get the observed reading involving an upper bound on the number of cards Cody is allowed to pick, it is customary to assume that the semantics of -er contains a maximality operator over degrees or cardinalities (and so, crucially, not a maximality operator over groups, like $\Sigma) .{ }^{15}$ So, rather than on $(74 b)$, the semantics of (74a) should be based on (75). The two readings for (74a) correspond to either having the modal within or outside the scope of the maximality operator, as in (76a) and (76b). (See Hackl 2000 for detailed discussion.)

$$
\max _{d}[\exists x[\operatorname{card}(x) \& \operatorname{pick}(c, x) \&|x|=d]]<6
$$
a. $\diamond \max _{d}[\exists x[\operatorname{card}(x) \& \operatorname{pick}(c, x) \&|x|=d]]<6$
b. $\max _{d}[\diamond \exists x[\operatorname{card}(x) \& \operatorname{pick}(c, x) \&|x|=d]]<6$

The problem is, now, that on this analysis any comparatively modified numeral is interpreted statically, given the wide scope of the modal and the maximality operator. Consequently, on the dynamic exhaustivity account fewer/more than three will wrongly be predicted to give rise to the same implicatures as no more/ fewer than four. This means that so far we are unable to have both dynamic exhaustification and an account of the comparative nature of comparatively modified numerals. In contrast, as I explained in previous sections, the UDM and the comparative semantics for modified numerals need no further reconciliation.

\footnotetext{
15 One might think the same effect can be derived by assuming a semantics as in (74b) without a form of maximality, but with the possibility of (minimal model style) exhaustive interpretation. Giving the modal wide scope over (74b) results in the weak reading that has been observed for (74a). Exhaustifying that reading does not yield the preferred interpretation, however. The reason is that (74b) with wide scope ' $\diamond$ ' is very weak: it does not pose any bound on how many card Cody is allowed to pick. Consequently, if we take the background question predicate to be the set of groups of cards Cody is allowed to pick, then the minimal worlds that verify (74b) will be worlds in which Cody isn't allowed to pick any card. If, rather than minimality, maximality is at stake, the worlds in which Cody is allowed to pick any number of cards will be selected, since these too are compatible with (74b).
} 
Somewhat related to this is the fact that the UDM can explain why implicatures suddenly do arise with more than numerals once they are embedded under strong modals. (This is one of the major points of Fox and Hackl 2006.) The example in (77) implicates that Cody is not required to pick more than five cards.

(77) Cody is required to pick more than four cards.

The implicature for (77) falls out of the UDM, since strong modals have the property of turning open scalar properties into closed ones (see Fox and Hackl 2006 for details). Crucially, weak modals lack this feature, which explains why (78) does not yield an implicature.

Cody is allowed to pick more than four cards.

If the contrast between weak and strong modals were to be explained in terms of dynamic exhaustivity, one would have to assume a dynamic profile for strong modals and a static one for weak modals. I can see no independent motivation for this.

\subsection{Alternatives}

Fox (2007) discusses an alternative to the Universal Density of Measurement assumption based on the observation that modified numerals like four or more do not yield implicatures. Fox links such cases to the simple disjunctions discussed by Sauerland (2004). A sentence like John talked to Mary or Sue is asymmetrically entailed both by John talked to Mary and by John talked to Sue, and so we might think that there are at least the implicatures that John did not talk to Mary and that John did not talk to Sue. However, such implicatures in tandem deny the assertion that was made. This, according to Sauerland, is why such implicatures do not arise.

Fox argues that a similar effect explains why four or more does not end up meaning exactly four. He notices first of all that such disjunctive modified numerals are in danger of violating a general constraint on disjunction known as Hurford's Generalisation (Hurford 1974):

(79) Hurford's Generalisation:

\#[A or B] whenever B entail A

Standard illustrations of this generalisation are examples like \#John lives in France or in Paris. If (79) is to apply to all disjunctions, then one might wonder why four or more marbles is felicitous, since the lower bound reading of the numeral is entailed by the second disjunct. Fox concludes that such modified numerals should therefore be seen as already partly locally exhaustified. So four or more corresponds to exh[four] or more. Exhaustification here will be similar to the cases discussed by Sauerland: (80a) yields two implicatures, (80b) and (80c), which taken together with (80a) form a contradiction, and this, Fox argues, is why no implicature arises. 
(80) a. Cody found four or more marbles.

b. It is not the case that Cody found exactly four marbles.

c. It is not the case that Cody found more than four marbles.

Such an explanation, Fox notes, at the same time paves the way for an alternative to the universal density approach, for, as suggested in Spector (2005), one could take more than three to have the same alternatives as four or more. So, Cody found more than three marbles has the implicatures in (80b) and (80c). Although the particular set of alternatives used for such an explanation has to be stipulated and is rather unintuitive, it would give all the right results with respect to exhaustification. (See Fox 2007 for detailed discussion. See also Russell 2006.)

Note, however, that such an alternative explanation becomes doubtful in the light of the observations I have presented in the previous sections, for it necessitates a further stipulation that no fewer than four differs from four or more and more than three with respect to its alternatives. To me, this seems to be one stipulation too many. In any case, I am extremely sceptical that we will find a reason (independent of the very observations made here) for both why the latter two share their alternatives and why the former's set of alternatives differs from these. This paper demonstrates the need to distinguish two classes of modified numerals, but I see no way of making it convincing that what sets these two classes apart is the set of alternatives the expressions in question give rise to.

\subsection{Conclusion}

The main point of the paper is a rather simple one: some modified numerals give rise to (scalar) implicatures. I have argued that one explanation for this is that such implicatures are the result of exhaustification of non-strict comparatives with respect to a dense scale. An approach involving dense scales has important advantages over alternative theories, since it predicts such a division, without further assumptions. One alternative to the use of the density assumption, however, is to counter my claim that the two classes correspond to the strict/non-strict comparison distinction and to assume that it is instead the negation in no -er constructions that is responsible for the implicatures. In that case, a theory of dynamic exhaustivity as in van Rooij and Schulz (2006) might be able to cover the data. Unfortunately, it is so far not clear how to extend such a theory so that it accounts for the range of data involving comparatives discussed in Fox and Hackl (2006) and Hackl (2000). Although the above is not an unequivocal demonstration of the role of density in the process of exhaustification, it shows that density covers the full set of data involving modified numerals with only minimal and independently developed means.

The data discussed in this paper sharpen the desiderata for theories of exhaustification. In this regard, the analysis illustrates the merits of conducting a detailed inquiry into the distinguishing features of various kinds of particular quantifiers, despite the fact that, on the surface, such quantifiers might not seem to host any interesting semantic mysteries. 
Open Access This article is distributed under the terms of the Creative Commons Attribution Noncommercial License which permits any noncommercial use, distribution, and reproduction in any medium, provided the original author(s) and source are credited.

\section{References}

Barwise, J., and R. Cooper. 1981. Generalized quantifiers and natural language. Linguistics and Philosophy 4(2): 159-219.

Bierwisch, M. 1989. The semantics of gradation. In Dimensional adjectives, ed. M. Bierwisch and E. Lang, 71-261. Berlin: Springer-Verlag.

Fox, D. 2007. Too many alternatives: density, symmetry and other predicaments. In Proceedings of SALT XVI, ed. M. Gibson and T. Friedman, Ithaca, NY: CLC Publications (forthcoming).

Fox, D., and M. Hackl. 2006. The universal density of measurement. Linguistics and Philosophy 29(5): 537-586.

Geurts, B. 2007. Experimental support for a modal analysis of at least and at most. In Unpublished Proceedings of the ESSLLI2007 Workshop on Quantifier Modification, ed. R. Nouwen and J. Dotlacil.

Geurts, B., and R. Nouwen. 2007. At least et al.: the semantics of scalar modifiers. Language 83(3): 533-559.

Hackl, M. 2000. Comparative quantifiers. PhD thesis, Department of Linguistics and Philosophy, Massachusetts Institute of Technology.

Heim, I. 2000. Degree operators and scope. In Proceedings of SALT 10, ed. B. Jackson and T. Matthews, 40-64. Ithaca, NY: CLC Publications.

Heim, I. 2006. Remarks on comparative clauses as generalized quantifiers. Ms., MIT.

Hurford, J. 1974. Exclusive or inclusive disjunction. Foundations of Language 11: 409-411.

Jespersen, O. 1949. A modern English Grammar on historical principles, part 2 syntax. København: Munksgaard.

Jespersen, O. 1966. Negation in English and other languages, Historisk-filologiske meddelelser / udg. af Det Kongelige Danske Videnskabernes Selskab, 2nd edn. København: Munksgaard.

Kamp, H., and U. Reyle. 1993. From discourse to logic. Dordrecht: D. Reidel.

Keenan, E., and J. Stavi. 1986. A semantic characterization of natural language determiners. Linguistics and Philosophy 9: 253-326.

Kennedy, C. 2001. Polar opposition and the ontology of 'degrees'. Linguistics and Philosophy 24: 33-70.

Krifka, M. 1999. At least some determiners aren't determiners. In The semantics/pragmatics interface from different points of view, Vol. 1 of Current research in the semantics/pragmatics interface, ed. K. Turner, 257-291. New York: Elsevier.

Nouwen, R. 2007. On dependent pronouns and dynamic semantics. Journal of Philosophical Logic 36(2): 123-154.

Nouwen, R. 2008a. Directionality in modified numerals: The case of 'up to'. In Proceedings of SALT 18, ed. T. Friedman and S. Ito. Ithaca, NY: CLC Publications (forthcoming).

Nouwen, R. 2008b. What's in a quantifier?. In Theoretical validity and psychological reality, ed. T. Lentz, H. de Mulder, Ø. Nilsen, and A. Zondervan. Amsterdam: Benjamins.

Rett, J. 2007. Antonymy and evaluation. In Proceedings of SALT 16 ed. M. Gibson and T. Friedman. Ithaca, NY: CLC Publications (forthcoming).

Russell, B. 2006. Against grammatical computation of scalar implicatures. Journal of Semantics 23: 361-382.

Sauerland, U. 2004. Scalar implicatures in complex sentences. Linguistics and Philosophy 27: 367-391.

Sawada, O. 2005. The cognitive characteristics of the idiomatic comparative constructions: the case of the no more/less...than constructions. In: Proceedings of the 9th Conference of the Pan-Pacific Assocation of Applied Linguistics, 273-280. Tokyo, Japan: Waseda University Media Mix Corp.

Spector, B. 2005. Aspects de la pragmatique des opérateurs logiques. PhD thesis, Université Paris 7.

Stoffel, C. 1894. Studies in English. Zutphen: W.J. Thieme \& Co.

van Benthem, J. 1986, Essays in logical semantics. Dordrecht: Reidel.

van Rooij, R., and K. Schulz. 2006. Pragmatic meaning and non-monotonic reasoning: the case of exhaustive interpretation. Linguistics and Philosophy 29: 205-250.

von Stechow, A. 1984. Comparing semantic theories of comparison. Journal of Semantics 3:1-77. 